\title{
MATERIALES NEOLITICOS EN EL VALLE DEL JARAMA (ARGANDA, MADRID)
}

\author{
POR \\ JULIO MERCADER FLORIN (*) \\ A. FRANCISCO CORTES BUSTOS (*) \\ M. ${ }^{a}$ EUGENIA GARCIA DE BENITO (*)
}

\begin{abstract}
RESUMEN En esta noticia se presentan materiales neolíticos del yacimiento del "Arenero de Arganda” (Madrid), constituyendo éstos una nueva aportación al panorama del Neolitico Interior.

Se propone, además, la necesidad de un replanteamiento en las estrategias explicativas que hasta ahora se han manejado en la interpretación del Neolítico meseteño.
\end{abstract}

\begin{abstract}
The Neolitihic materials from the site of "Arenero de Arganda" (Madrid), constitute an interesting addition to our knowledge of the Neolithic of the interior of the Iberian Peninsula. The materials suggest the need for a revision of the prevailing explanations of the nature of this Neolithic facies.
\end{abstract}

Palabras clave Neolítico Interior, Terraza, Arganda-Madrid, Modelos Explicativos.

\section{INTRODUCCION}

Los materiales de esta noticia están depositados en los fondos del Museo Arqueológico Nacional (1) habiendo sido publicados parcialmente en dos ocasiones (Antona, 1986; Idem 1987) sin que en ninguna de ellas se documentasen formas, rasgos de las piezas, etc. Precisamente como complemento a la información ya existente presentamos nuevos datos sobre los hallazgos de este enclave neolítico del interior peninsular.

() Lcdos. en Geografía e Historia (especialidad de Prehistoria). Facultad de Geografía e Historia. Universidad Complutense. Madrid.

(1) Tuvimos acceso a estos materiales gracias a C. Cacho y M. ${ }^{\mathrm{a}}$ I. Martínez Navarrete. Nuestro agradecimiento a ambas, y muy especialmente a la última por la lectura crítica del original. Igualmente a T. Sanz por habernos facilitado la consulta de los mismos. 


\section{LOCALIZACION Y CARACTERISTICAS DEL HALLAZGO (2)}

En el año 1976 M. Santonja realizó excavaciones en los yacimientos de Aridos I y II en las proximidades de Arganda (Madrid), muy cerca de la confluencia del Manzanares con el Jarama. En el transcurso de las mismas recibió de D. Francisco Fernández Correas (Empresa Aridos, S. A.) materiales recuperados mientras se extraian arenas un kilómetro al norte, aproximadamente, del lugar en el que se excavaba (Fig. 1).

Hemos podido averiguar muy poco del contexto de los hallazgos. Sabemos que aparecieron todos en un mismo punto y en los "niveles superiores" de la terraza. Ignoramos si estaban o no asociados a estructuras y si éstos sufrieron selección.

\section{LOS MATERIALES}

. Además de los fragmentos cerámicos dibujados (Fig. 2 y 3), en el lote conservado existen piezas de difícil asignación pero que fueron recogidas junto con las demás. Nos referimos a dos «manos de molino" con "pulimento" y a una veintena, aproximadamente, de fragmentos cerámicos atípicos.

Las cerámicas fueron realizadas a mano. El grosor medio de la pared oscila entre 0,6 y $0,8 \mathrm{~cm}$. Los colores de las pastas son sombras, sienas, negros y grises. Los desgrasantes utilizados son cuarzo, mica y cal, de tamaño fino $(1-2 \mathrm{~mm}$.), medio $(2-3 \mathrm{~mm}$.) y grueso $(3-4 \mathrm{~mm}$.). Las cocciones son reductoras o alternantes y la estructura de la pasta variable (compacta, foliar y disgregada). El color de las superficies es siena, salvo en un caso con superficies interiores oscuras. Los tratamientos superficiales son alisados al interior y al exterior, of reciendo la vasija de la Fig. 3 la peculiaridad de tener gran cantidad de surcos en direcciones variables sobre la cara interior (¿alisado con entramado vegetal?). Alguna superficie está erosionada y casi todas ellas tienen concrecciones.

\section{CONCLUSION}

Aunque tipológicamente los materiales ofrecen semejanza con los de grupos de otras regiones, encuadrables en un momento relativamente avanzado del Neolítico Peninsular, no somos partidarios de proporcionarles una-asignación cronológico-cultural concreta, basándonos solamente en los paralelos formales. Además, los estudios de recogidas superficiales y de fondos antiguos depositados en la Sección Arqueológica del Museo Municipal de Madrid (3), nos han permitido observar la dificultad que conlleva la aceptación a priori de modelos explicativos constatados en otras áreas, pero no en la Región Centro.

Así, pues, las estrategias manejadas hasta el momento pueden ponerse en duda (Mercader et alii, en prensa), y muy especialmente en lo concerniente a la cuestión del asentamiento y sus implicaciones culturales, cronológicas y económicas.

Fue Pérez de Barradas el primero en proponer la existencia del «Neolítico» en la Región Central (Pérez de Barradas, 1926), defendiendo la existencia de numerosas ocupaciones al aire libre con estructuras de hábitat llamadas «fondos de cabaña» (Pérez de Barradas, 1929: 302-308).

La caracterización efectuada por este autor fue aceptada hasta la década de los 70 , en la que la

(2) Agradecemos desde aqui a M. Santonja y A. Méndez los datos facilitados acerca de las condiciones en las que se produjo el descubrimiento de los materiales.

(3) Nuestro agradecimiento a M. a C. Priego y S. Quero por las facilidades prestadas en la consulta de fondos del Museo de la Fuente del Berro desde diciembre de 1986. 

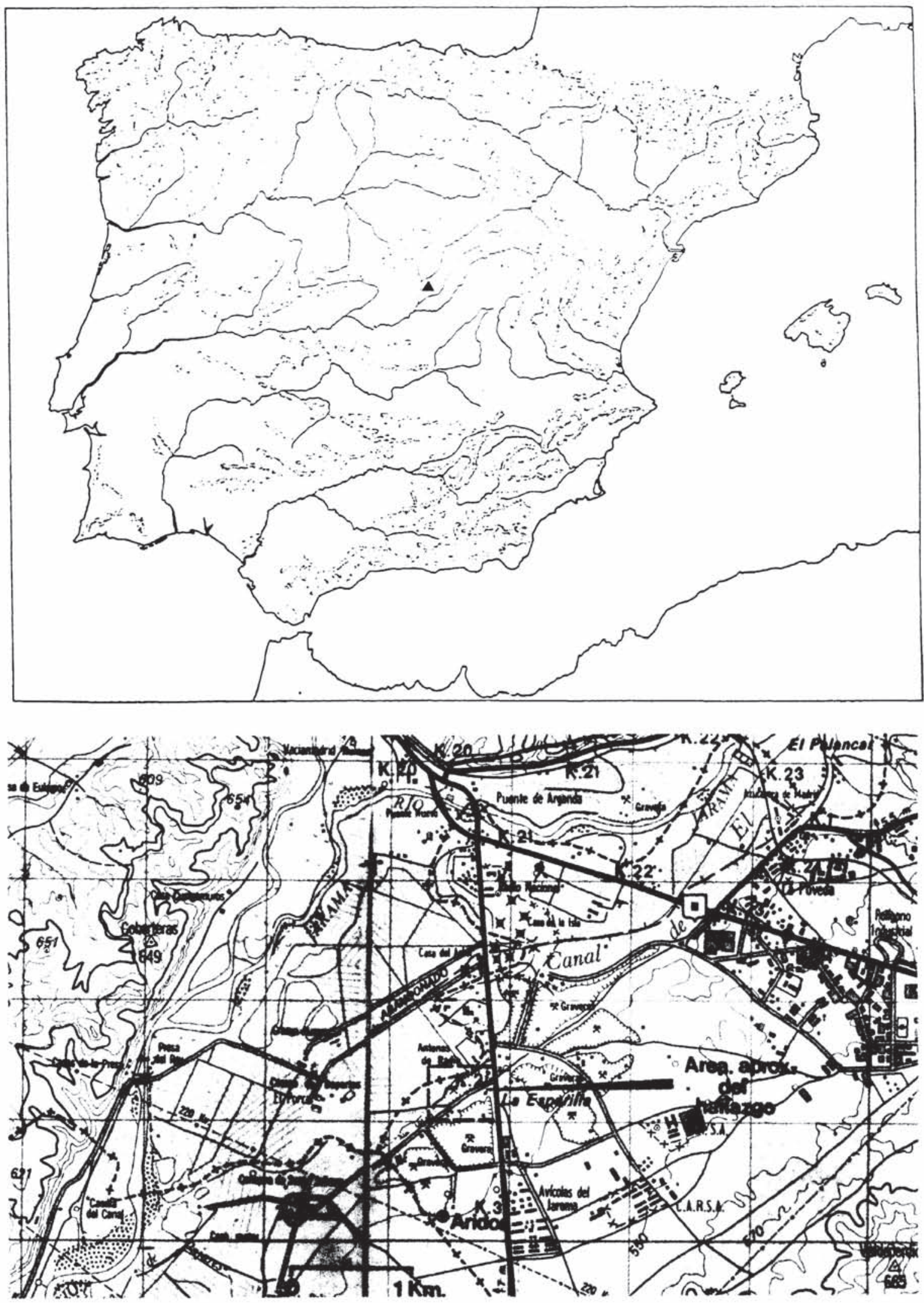

Fig. 1.- Situación del yacimiento en la Península Ibérica y en el M. T. N. del S. G.E., E 1:50.000. hojas núms. 582 («Getafe») y 583 («Argandcos). 

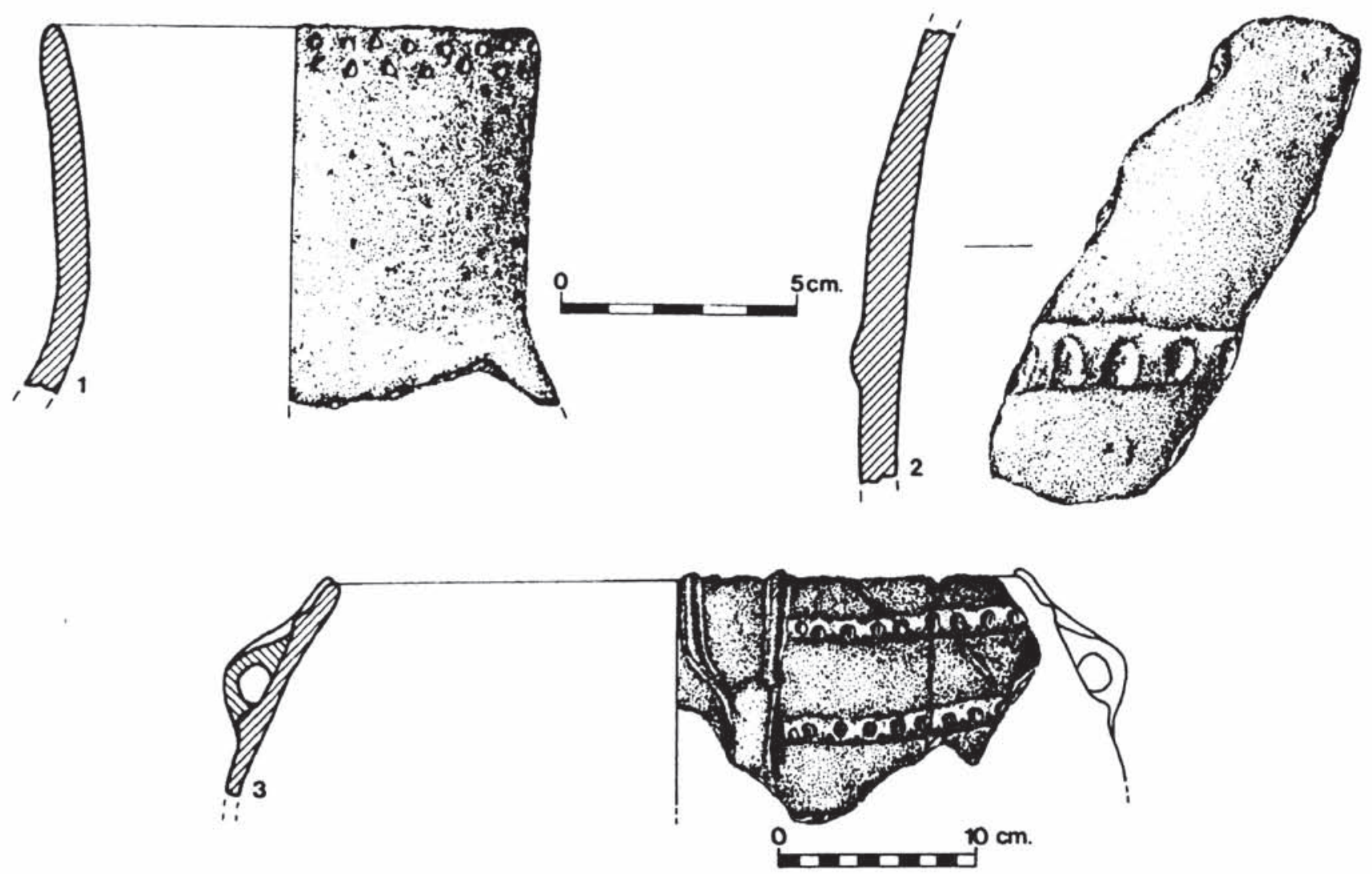

FIG. 2.- Boca de botella, fragmento de pared y vasija de paredes entrantes.
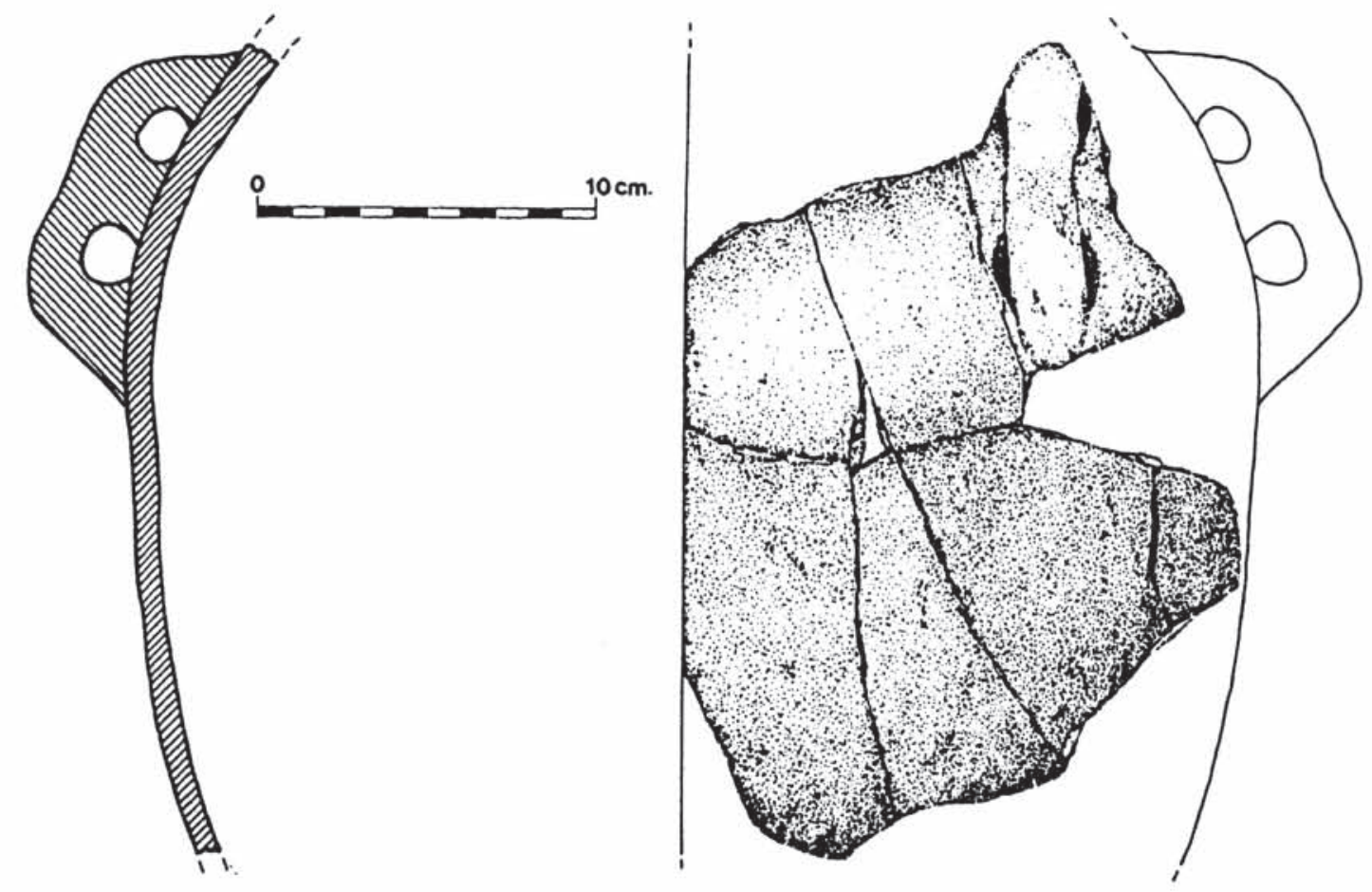

FIG. 3.- Reconstrucción de vasija de gran tamaño. 
revisión de parte de los materiales clasificados como "neolíticos" por el investigador clásico demostró que no había bases suficientes para defender tal filiación (López, 1977: 372; también en Martínez Navarrete, 1979: 108).

En 1980, M. ${ }^{a}$ D. Fernández-Posse publicaba un lote de materiales de la Cueva del Aire, planteando de nuevo la presencia de gentes neolíticas en el territorio madrileño, volviendo "pendularmenten con ello al postulado «clásico". Ahora bien, en esta ocasión el antecedente no era Pérez de Barradas, sino Bosch Gimpera, obviando completamente los presupuestos del primero y delimitando como principales características la procedencia meridional de los grupos, su carácter tardío y el hábitat en cuevas de los sistemas montañosos marginales (Fernández-Posse, 1980).

Sin embargo, hoy por hoy, la mayoria de asentamientos conocidos son al "aire libre" y se sitúan en la Fosa del Tajo. Con ello no afirmamos tanto el carácter mayoritario de este patrón (en la medida en que no se han llevado a cabo investigaciones en la totalidad del territorio), como la no conveniencia de manejar apriorismos. Por otro lado, tampoco se olvide que las formaciones kársticas en la provincia se circunscriben a pequeños terrenos del NE y SE.

Se dijo también que los enclaves al aire libre eran más modernos que los de cueva, utilizando argumentos evolutivos - bien de cariz socio-económico (Delibes, 1985: 26), bien tipológicos (Antona, 1987: 53) - constatados en otras áreas (Arribas y Molina, 1979: 124) pero no en el interior. Si bien estas explicaciones podrían parecer inevitables, no son convenientes, pues según el estado actual de la cuestión ignoramos cuál es la relación real con las áreas periféricas y la reacción del sustrato - que obviamente tuvo que existir - ante el proceso de neolitización, ya que la similitud observada entre los materiales de unas y otras zonas no aclara, por el momento, más que la pertenencia a una misma dinámica general y no un avance o colonización. Desconocemos también el comportamiento socio-económico de los grupos y no observamos en los materiales sin estratigrafías hasta ahora conocidos ningún desarrollo tipológico-contextual que abogue por fechas más tardías para los yacimientos al aire libre (cf. Antona, 1986: 29; Idem, 1987: 53).

Igualmente, sigue siendo problemático el averiguar como eran las estructuras a las que estaban asociados los materiales clásicos y de superficie y si éstos pueden describirse o no como «fondos de cabañan, pues esto es evidente sólo en ciertos casos. Serian posibles fórmulas alternativas que habrá que constatar o desechar.

Parece clara, además, la posibilidad de poner en entredicho la tan defendida escasa entidad de la ocupación neolítica de estos territorios, pues cada dia son más los yacimientos descubiertos y es seguro que los trabajos futuros de investigación podrán localizar nuevos lugares y aproximarse más a esta etapa tan importante de la Prehistoria regional.

\section{BIBLIOGRAFIA}

ANTONA, V. (1986): «Aproximación a la problemática del Neolítico en la Meseta: Una propuesta de secuencia culturaln. W. A. H. 13. pp. 9-43.

- (1987): «El Neolítico» en 130 años de arqueología madrileña. Madrid. pp. 44-57.

Arribas, A. y Molina, F. (1979): «El poblado de los Castillejos en Las Peñas de los Gitanos (Montefrío, Granada). Campaña de excavaciones de 1971» C. P. U. G. Serie monográfica, $\mathrm{n}^{2} 3$.

BARRIO, J. (en prensa): «El yacimiento neolítico del Covacho de la Higuera (Patones, Madrid).

DELIBES DE CASTRO, G. (1985): «El Neolítico. Los comienzos de la agricultura y la ganaderia en la Meseta» en $\mathrm{La}$ Prehistoria del Valle del Duero. H.a de Castilla y León. Vol. I. Valladolid. Ed. Ambito. 1985. pp. 22-35.

FernánDeZ-POSSE, M.a D. (1980): «Los materiales de la Cueva del Aire de Patones (Madrid)» N. A. H. Preh. $\mathrm{n}^{2} 3$. pp. $189-236$.

LORIANA, M. DE (1942): «Nuevos hallazgos del Vaso Campaniforme en la provincia de Madrid» A. E A. XV pp. 161167. 
López, P. (1978): El Neolitico en la Peninsula Ibérica. Perspectivas actuales de su estudio. (Tesis doctoral) U. C. M. 2 vols. Inédita.

LOSADA, H. (1976): «El dolmen de Entretérminos (Madrid)» T. P. 33 (Nueva Serie). pp. 209-226.

Martínez Navarrete, M. ${ }^{a}$ I. (1979): «El yacimiento de 'La Esgaravita' (Alcalá de Henares, Madrid) y la cuestión de los llamados 'fondos de cabaña' del valle del Manzanaresw. T.P. $\mathrm{n}^{2}$ 36. pp. 83-118.

Méndez, A. y Gálvez. P. (1984): «Nuevos materiales de la Edad de Bronce en el término de Madrid. El yacimiento del km. 3,5 Izquierda de la carretera de San Martin de la Vegan. E P. A. M. pp. 33-73.

Mercader, J. et alii (en prensa): «Nuevos yacimientos neoliticos y de la Edad del Bronce en el término municipal de Madrid $E$. P. A. M.

Pérez de Barradas, J. (1926): «El Neolítico de la provincia de Madrid» R. B. A.M. A. M. III pp. 75-87.

- (1929): «Los yacimientos prehistóricos de los alrededores de Madrid» B. I. G. M. E. T. LI/T. XI (3.a Serie) pp. 153-322.

- (1933): «Nuevos estudios sobre Prehistoria madrileña. I. La Colección Bento». A. P. M. vol. IV-V-VI. pp. 1-90.

- (1941): „Poblado Prehistórico de Los Vascos (Villaverde, Madrid)». A. M. S. E A. E. P. T. XVI. Cuadernos I y II. pp. 158-160. 